\title{
New 2-D Adaptive K-Best Sphere Detection for Relay Nodes
}

\author{
Ahmad El-Banna ${ }^{* 1,2}$ \\ ${ }^{1}$ Electrical Eng. department, Faculty of Engineering at Shoubra, Benha Univ., Egypt. \\ ${ }^{2}$ ECE department, Egypt-Japan University of Science and Technology (E-JUST), Alexandria, Egypt
}

\begin{abstract}
Relay nodes are the main players of cooperative networks that used to improve the system performance and to offer virtual multiple antennas for limited antenna devices in a multi-user environment. However, employing relaying strategies requires considerable resources at the relay side and places a large burden on the relay helping node especially when considering the power consumption. Partial detection at the relay is one strategy that reduces the computational load and power consumption. In this paper, we propose a new 2-D Adaptive KBest Sphere Decoder (2-D AKBSD) for partial detection to be used MISO relays in cooperative networks. Simulation results show that 2-D AKBSD is capable of improving the system performance and also reduces its complexity.
\end{abstract}

Keywords-Sphere Detection; K-Best; Relay; MIMO; MISO; Cooperative Communication

\section{INTRODUCTION}

Cooperative networks enhance the performance of the communication system by increasing the transmission data rate making use of the cooperative diversity property. It removes the need for installing multiple antennas at the network nodes which may be in many cases impractical due to size, hardware complexity and/or power limitations [1]-[6]. Cooperative networks can also be used to extend the network coverage area and to reduce the transmit power [7].

To utilize the cooperative networks merits, cooperation protocols need to be employed, two main protocols have been widely adopted for relaying. The first one is "Amplify and Forward" (AF) in which the received signal is amplified at the relay and then forwarded to the destination. The second one is "Detect" or "Decode and Forward" (DF) in which the received signal is detected and then decoded and forwarded to the destination. DF gives better performance than the simpler AF scheme, a property that made DF the favorable relaying protocol for multihop system suffering from incremental loss of performance per hop [7]. However, DF requires more processing and consumes considerable resources at the relay. Hence, the relay consumed power is increased causing a problem in many cases, e.g. an idle mobile MIMO user may not choose to operate as a relay in DF mode due to its limited battery power.

Partial Detection (PD) at the relay can reduce the complexity of the decoding by detecting only part of the received signal, depending on its available resources, then forwarding it. The destination combines both the source signal and the partial relay signal to recover the transmitted signal [8]. However, PD performance is poor when the number of the detected symbols at the relay is small [7], [8]. The PD in [8] modifies the tree search of the tree-based sphere detector depending on the relay available resources to include only some levels of the tree instead of all tree levels in the case of Full Detect and Forward (FDF); we name this a change in the number of vertical levels in a tree search.

The partial sphere detection scheme proposed in [8] was employed for the spatial multiplexing transmission technique in a MIMO wireless communication. In this work, we utilize it for transmit antenna diversity techniques by employing the Alamouti and the Golden codewords in a time varying environment.

In this paper, we propose a new idea that allows a change in the two dimensions of the tree during the tree traversal to give more complexity reduction at the relay node. The first dimension is the horizontal dimension, in which we change the number of the visited nodes per level depending on some criteria [9] e.g. the channel quality. The second dimension to change is the vertical dimension in which the number of the explored tree levels is chosen depending on the source-relay link capacity, besides this change is adaptive depending on the current state of the network environment specifically the channel and link qualities in the network. The proposed detector, namely 2-D AKBSD, gives a considerable complexity reduction in the relay and destination nodes.

The rest of the paper is organized as follows; Section II describes the network and system model of the $2 \times 1$ MISO scenarios that was used to verify the 2-D AKBSD. Section III illustrates how the 2-D AKBSD can be used as the decoding strategy in the $2 \times 1$ relay. Section IV shows the simulation results and finally, section $\mathrm{V}$ concludes the paper.

\section{NETWORK AND SYSTEM MODELS}

Following the same system model notations in [8], we consider a 3 nodes wireless network with one Source node (S) that cooperate with one Relay node (R) assisting in data transmission to one Destination node (D). The distance between the $\mathrm{S}$ and $\mathrm{D}$ nodes is denoted as $d_{s d}$ while $d_{s r}$ and $d_{r d}$ define the distances between the S-R and R-D nodes respectively. Each node is equipped with $N_{t}$ and $N_{r}$ transmitting and receiving antennas respectively. We studied the MISO case with $N_{t}=2$ and $N_{r}=1$. The transmission is assumed to be executed over two phases $\mathrm{T}_{1}$ and $\mathrm{T}_{2}$. We assume a half-duplex communication i.e. nodes can only transmit or receive at the same instance, and consider a Rayleigh time-varying fading channel, a case where linear 
decoding is no more the optimum decoding method as in the quasi-static channel case and more sophisticated decoding algorithms are required to obtain a near optimum performance. We refer to the channels between $S$ and $D, S$ and $R$ and $R$ and $\mathrm{D}$ as $\mathbf{H}_{\mathrm{sd}}, \mathbf{H}_{\mathrm{sr}}$ and $\mathbf{H}_{\mathbf{r d}}$ respectively and it is assumed that these channels are known in their corresponding receiving nodes while the signal-to-noise ratios (SNRs) at the nodes receiving antennas are defined as [8]

$$
S N R_{s d}=\frac{\mu p}{\left(d_{s d}\right)^{\alpha}}, S N R_{s r}=\frac{\mu p}{\left(d_{s r}\right)^{\alpha}}, S N R_{r d}=\frac{(1-\mu) p}{\left(d_{r d}\right)^{\alpha}}
$$

where $p$ is the system total transmit power and is split among the source and relay nodes using the factor $\mu$ where $0<\mu<1$, while $\alpha$ is the path loss exponent and its value is usually chosen between 2 and 6 . We employed the Alamouti codeword as Space Time Block Codes (STBC) for the transmit diversity technique at the transmitting node. The Alamouti $(\mathbf{X})$ codeword is defined as

$$
\boldsymbol{X}=\left[\begin{array}{cc}
x_{i} & x_{i+1} \\
-x_{i+1}{ }^{*} & x_{i}{ }^{*}
\end{array}\right],
$$

respectively where (.)* denote conjugate operation, $x_{i}$ represents the $i^{\text {th }}$ transmitted symbol. The system and the received signals model are modeled as follows.

The nodes are equipped with two transmit antennas and one receive antenna as shown in Fig. 1, and it is assumed that $\mathrm{R}$ node uses the codeword $\mathbf{X}$ in its transmission during the second phase of communication, transmission from $\mathrm{S}$ and $\mathrm{R}$ nodes through the $\left(t_{i}, i=1: 4\right)$ four time instants frame of $\mathrm{T}_{1}$

\begin{tabular}{|c|c|c|c|c|c|c|c|}
\hline \multicolumn{5}{|c|}{$t_{1}$} & \multicolumn{3}{|c|}{$t_{2}$} \\
\hline \multicolumn{3}{|c|}{$\underbrace{S}$} & \multicolumn{2}{|l|}{$R$} & \multicolumn{2}{|l|}{$S$} & $R$ \\
\hline$\overbrace{t x_{i}}^{x_{i}}$ & & $\frac{c_{i+1}^{*}}{x_{2}}$ & $\overbrace{t x_{1}}^{0}$ & $\begin{array}{r}\underbrace{0}_{t x_{2}} \\
\mathrm{~T}_{2}\end{array}$ & $\overbrace{t x_{1}}^{x_{i+1}}$ & $\underbrace{x_{i}^{*}}_{t x_{2}}$ & $\overbrace{t x_{1}}^{0} \underbrace{0}_{t x_{2}}$ \\
\hline & & $t_{3}$ & & & & $t_{4}$ & \\
\hline$S$ & & & $\underbrace{R}$ & & $\underbrace{S}$ & & $\underbrace{R}$ \\
\hline$\underbrace{0}_{t x_{1}}$ & $\underbrace{0}_{t x_{2}}$ & $\underbrace{\hat{x}_{i}}_{t x_{1}}$ & $\underbrace{-\hat{x}_{i}}_{t x}$ & & $\underbrace{0}_{t x_{1}} \underbrace{0}_{t x}$ & $w_{x_{2}}^{0} \underbrace{\hat{x}}_{t}$ & $\underbrace{i+1}_{t x_{1}} \underbrace{\hat{x}_{i+1}}_{t x_{2}}$ \\
\hline
\end{tabular}
and $\mathrm{T}_{2}$ is described as

where $t x_{1}$ and $t x_{2}$ are the $1^{\text {st }}$ and $2^{\text {nd }}$ transmitting antenna of $\mathrm{S}$ or $\mathrm{R}$ node. This communication protocol needs 4 channel uses to send 2 symbols giving a symbol rate of 0.5 symbol per channel use (symb. pcu).

As shown in Fig. 1 and assuming the sending node is transmitting a MISO $2 \times 1$ Alamouti diversity code, the relay node and the single antenna destination node receive the source signal using one antenna. This means that the relay uses only one antenna for the reception of the source data while the other antenna is idle or off. In the transmission phase, the relay uses its two antennas to transmit the $2 \times 1$ signal to the destination node in the relaying phase.

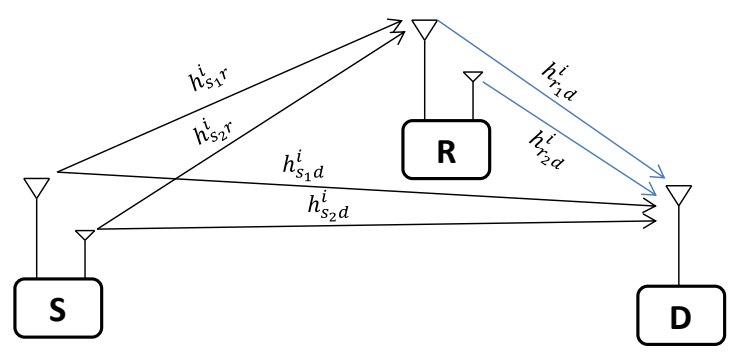

Fig. 1. S-R-D network with $2 \times 1$ nodes

The received signals at $\mathrm{R}$ and $\mathrm{D}$ nodes in the first two phases are

$$
\begin{array}{rr}
\text { R: } & \mathrm{T}_{1}\left\{\begin{array}{l}
y_{r}^{1}=h_{s_{1} r}^{1} x_{1}-h_{s_{2} r}^{1} x_{2}{ }^{*}+n_{1} \\
y_{r}^{2}=h_{s_{1} r}^{2} x_{2}+h_{s_{2} r}^{2} x_{1}{ }^{*}+n_{2}
\end{array}\right. \\
\mathbf{D :} & \mathrm{T}_{1}\left\{\begin{array}{l}
y_{d}^{1}=h_{s_{1} d}^{1} x_{1}-h_{s_{2} d}^{1} x_{2}{ }^{*}+v_{1} \\
y_{d}^{2}=h_{s_{1} d}^{2} x_{2}+h_{s_{2} d}^{2} x_{1}{ }^{*}+v_{2}
\end{array}\right. \\
\mathrm{T}_{2}\left\{\begin{array}{l}
y_{d}^{3}=h_{r_{1} d}^{1} \hat{x}_{1}-h_{r_{2} d}^{1} \hat{x}_{2}{ }^{*}+w_{1} \\
y_{d}^{4}=h_{r_{1} d}^{2} \hat{x}_{2}+h_{r_{2} d}^{2} \hat{x}_{1}{ }^{*}+w_{2}
\end{array}\right.
\end{array}
$$

where $h_{s_{k} d}^{i}, h_{s_{k} r}^{i}$ and $h_{r_{k} d}^{i}$ are the $i^{\text {th }}$ coefficients of the channels between the $k^{\text {th }}$ transmitting and the receiving antenna between $\mathrm{S}$ and $\mathrm{D}, \mathrm{S}$ and $\mathrm{R}$ and $\mathrm{R}$ and $\mathrm{D}$ respectively. Assuming $\hat{x}_{i}=x_{i}$, the combined two phases signals at the D node are

$$
\left\{\begin{array}{c}
y_{d_{1}}^{t}=\left(h_{s_{1} d}^{1}+h_{r_{1} d}\right) x_{1}-\left(h_{s_{2} d}^{1}+h_{r_{2} d}^{1}\right) x_{2}{ }^{*}+\psi_{1} \\
y_{d_{2}}^{t}=\left(h_{s_{2} d}^{2}+h_{r_{2} d}^{2}\right) x_{1}{ }^{*}+\left(h_{s_{1} d}^{2}+h_{r_{1} d}^{2}\right) x_{2}+\psi_{2}
\end{array}\right.
$$

where $\psi_{i}$ is the total AWGN noise at the $i^{\text {th }}$ phase.

\section{2-D AKBSD}

K-Best Sphere Decoder (KBSD) is a tree search method of the sphere decoding algorithm with a search in the forward direction only. The main advantage of the KBSD is its fixed throughput which makes it suitable for parallel and pipelined implementations; it also gives considerable complexity reduction. However, its Bit Error Rate (BER) performance is highly dependent on its $\mathrm{K}$ value which determines how many nodes will be visited while traversing the tree [8], [9]. Therefore, selecting the $\mathrm{K}$ value is a challenge as increasing it decreases the BER but increases the decoder complexity and vice versa. Adapting the $\mathrm{K}$ value depending on some criteria that measure the channel quality e.g. [9] is a solution for finding the optimum $\mathrm{K}$ value that best suits the varying conditions of the channel. This type of adaptation is performed over the horizontal level in the tree to achieve the best tradeoff between performance and complexity. In cooperative networks, another dimension of adaptation can be used within the KBSD search strategy e.g. [8] in which a partial detection is performed to partition the detection task between the $\mathrm{R}$ and $\mathrm{D}$ nodes, this partial detection employs the adaptation over the vertical levels of the tree depending on the available resources in the R node to reduce the overhead introduced by the MIMO relay. 


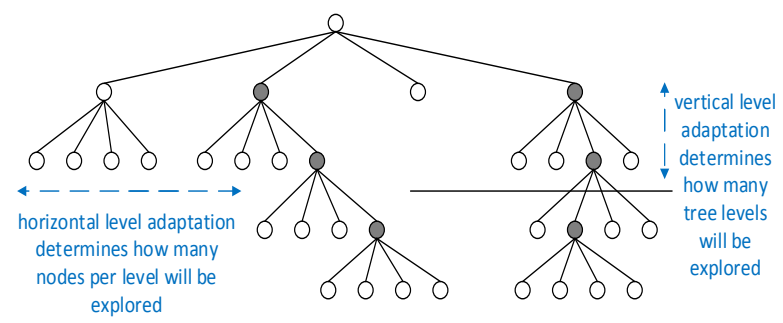

Fig. 2. Tree structure for 2-D AKBSD shows the horizontal and vertical adaptation

The 2-D AKBSD makes use of the two vertical and horizontal dimensions of the adaptation process in the $\mathrm{R}$ node to suit the dynamic conditions of the network. As shown in Fig.2, some criteria specify how many tree levels will be explored and how many nodes per level will be visited while traversing the tree. Adaptation criterion in horizontal dimension can be selectivity of the channel or channel matrix analysis as measures of channel quality i.e. selecting low or high $\mathrm{K}$ value depending on the quality of the channel or an estimation of SNR value as in [9] while vertical dimension adaptation can be done based on available resources in the relay as in [8], received signal strength or the instantaneous capacity measurement as a measure of the S-R link outage. In this paper, we select the channel selectivity as a channel quality estimation and the instantaneous capacity measurement as the adaptation criteria for the horizontal and vertical dimensions respectively. To measure the channel selectivity, we calculate a $\rho$ parameter as [9]

$$
\rho_{i}=\frac{\min \left(h_{i, j}, h_{i, j+1}\right)}{\max \left(h_{i, j}, h_{i, j+1}\right)}
$$

where $h_{i, j}$ and $h_{i, j+1}$ are two adjacent coefficients of the time-varying channel $i$ between any two nodes in the network. And if $C_{i}$ is greater than a defined threshold $\Gamma$ then the channel is very frequency selective and hence the channel quality is good and vice versa. The instantaneous capacity can be used as one metric that indicate the channel state information as a measure for the S-R link quality and can be calculated as [10]

$$
C=\log _{2} \operatorname{det}\left(\boldsymbol{I}_{N_{r}}+\frac{E_{x}}{N_{t} N_{0}} \boldsymbol{H}_{s r} \boldsymbol{H}_{s r}^{H}\right)
$$

where $E_{x}$ is the transmitted signal energy and $N_{0}$ is the noise power spectral density.

The 2-D AKBSD adds a pre-process part over the regular KBSD and hence it has the advantages of the KBSD, besides the advantage of being adapted based on the system dynamic conditions. The algorithm of the 2-D AKBSD is as follows:

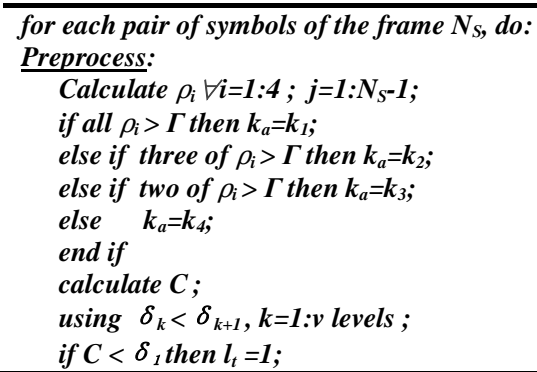

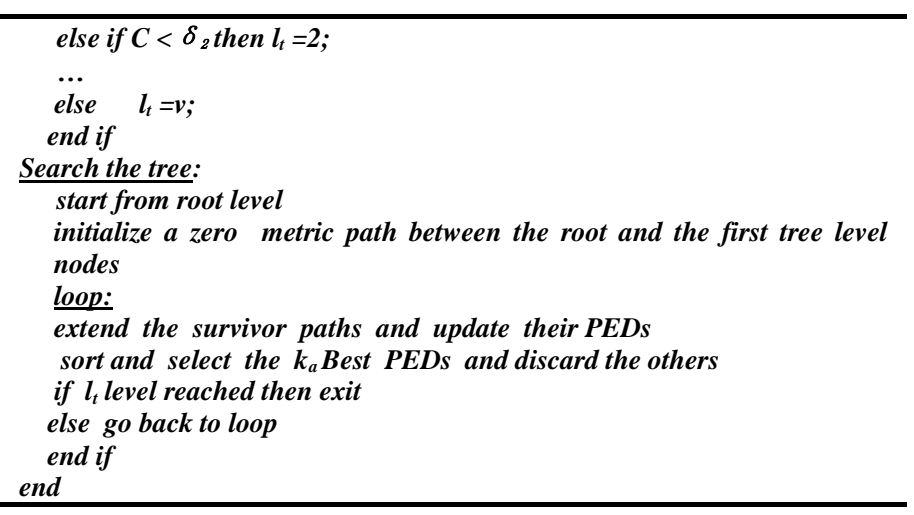

A. The detection strategy

For conventional FDF, both R and D nodes performs full detection of all the symbols using regular KBSD while for PD, the $\mathrm{R}$ node applies the 2-D AKBSD to detect part of the transmitted symbols, this part is determined based on the S-R link capacity, and in $\mathrm{T}_{2}, \mathrm{R}$ transmits the detected signals to the D node which uses 2-D AKBSD with horizontal adaptation only to retrieve the original signals.

\section{B. Single antenna relay in MISO environment}

A major advantage of the partial sphere detection is using a single antenna relay in a MISO transmission, using the codeword sent from the $\mathrm{S}$ node, $\mathrm{R}$ node can make horizontal adaptation and fix the vertical levels traversing to the half of the tree levels to send only half the symbols by its single antenna. In this manner, we can make use of an idle single antenna device to assist in data transmission in a $2 \times 1 \mathrm{MISO}$ protocol as shown in Fig. 3. This lifts the constraints of only involving multiple antennas relays in the transmission. Moreover, this will increase the data rate as we need only 3 channel uses instead of 4 giving symbol rate of $2 / 3$ symb. pcu.

\begin{tabular}{|c|c|c|c|}
\hline Phase & \multicolumn{2}{|c|}{$\mathrm{T}_{1}$} & $\mathrm{~T}_{2}$ \\
\hline $\begin{array}{c}\text { Time } \\
\text { Slot }\end{array}$ & $t_{1}$ & $t_{2}$ & $t_{3}$ \\
\hline $\begin{array}{c}\text { Transmit } \\
\text { Node }\end{array}$ & $\mathrm{s}$ & $\mathrm{S}$ & $\mathrm{R}$ \\
\hline$t x_{1}$ & $x_{i}$ & $x_{i+1}$ & $\hat{x}_{i}$ \\
\hline$t x_{2}$ & $-x_{i+1}{ }^{*}$ & $x_{i}{ }^{*}$ & - \\
\hline
\end{tabular}

Fig. 3. Single antenna relay in $2 \times 1 \mathrm{MISO}$ protocol

\section{Simulation RESUlTS}

We suppose half-duplex communication in a 3 node wireless relay network topology as shown in Fig. 1. We assume that the $\mathrm{R}$ node is in-between the $\mathrm{S}$ and $\mathrm{D}$ nodes with $d_{s d}=d_{s r}+d_{r d}=1$, fixing $d_{s r}=0.35$, and also we used fixed values of $\alpha=3, \mu=0.6$ and $p=1$. We work on 16-QAM modulation scheme and assumed that $\mathbf{H}_{\text {sd }}, \mathbf{H}_{\text {sr }}$ and $\mathbf{H}_{\text {rd }}$ are Rayleigh time-varying fading channels i.e. their coefficients are not constant during the transmission of the codeword elements as in the case of quasi-static channels. Monte Carlo simulations were used to calculate the BER values in the $R$ 
and D nodes besides calculating the complexity of FDF and 2-D AKBSD/PD decoding algorithms in terms of average number of visited nodes. The mean values of the instantaneous capacity were calculated and its immediate value was used to be checked against a threshold value and as a result a suitable number of levels of the vertical adaptation was selected as illustrated in the algorithm before. The threshold values $\delta$ and $\Gamma$ were chosen based on numerical analysis and their values are $\delta=3.5$ while $\Gamma=0.82$.

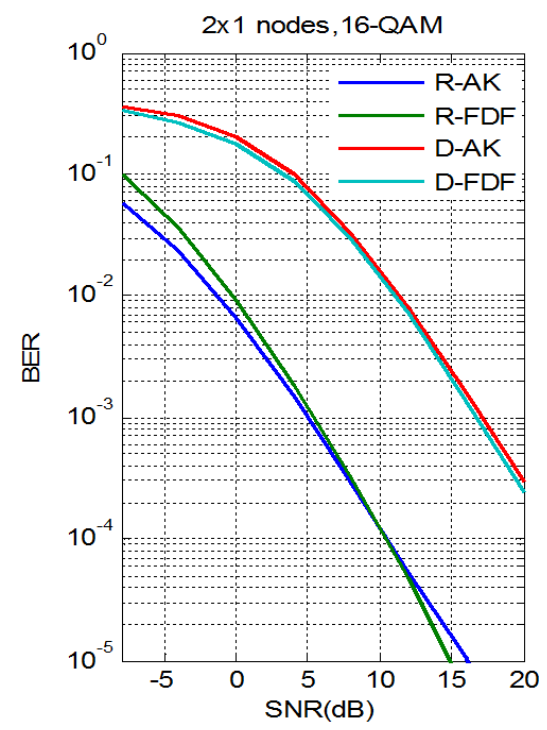

Fig. 4. BER performance for $2 \times 1$ nodes for $2 \mathrm{DAKBSD}$ and FDF detection strategies at $R$ and $D$ nodes

Figure 4 shows the BER performance of the $2 \times 1$ scenario for the FDF and 2-D AKBSD detection strategies at R and D nodes. PD using 2-D AKBSD shows a near FDF performance with a maximum loss of $0.47 \mathrm{~dB}$ all over the BER/SNR asymptotes. The worst asymptotic loss in performance is $0.47 \mathrm{~dB}$.

Complexity of each detection strategy of FDF and 2-D AKBSD was calculated in terms of average number of visited nodes and is summarized with the reduction percentage in $\mathrm{R}$ and D nodes in table $\mathrm{I}$; the range of reduction is $24.3 \%$ at the $\mathrm{D}$ node while at the $\mathrm{R}$ node is $22.2 \%$ which gives a system reduction at both the $\mathrm{R}$ and $\mathrm{D}$ together of $23.25 \%$. This computation complexity reduction is in turn a power reduction with the same percentage in the network devices consumed power.
TABLE I. COMPLEXITY MEASUREMENT

\begin{tabular}{|l|l|l|l|l|}
\hline FDF & \multicolumn{3}{|l|}{ 2-D AKBSD } \\
\hline (R \& D nodes) & $\begin{array}{l}\text { R } \\
\text { node }\end{array}$ & $\begin{array}{l}\text { \% } \\
\text { reductio } \\
\text { n }\end{array}$ & $\begin{array}{l}\text { D } \\
\text { node }\end{array}$ & $\begin{array}{l}\text { \% } \\
\text { reducti } \\
\text { on }\end{array}$ \\
\hline 37 & 27.97 & $24.4 \%$ & 28.8 & $22.2 \%$ \\
\hline
\end{tabular}

\section{CONCLUSIONS}

In this paper we propose a new 2-D adaptive K-Best sphere detector that reduces the complexity of both of the relay and destination nodes in cooperative wireless relay networks. The 2-D AKBSD performs horizontal and vertical adaptation processes while traversing the tree. The proposed algorithm adapts vertically the number of the tree levels depending on the source-relay link capacity as well as adapting horizontally the number of visited nodes per each level depending on the channel quality. This double adaptation saves considerable amount of processing, especially in the relay node, which in result saves the consumed power of the network nodes which is an important issue in modern wireless communication devices especially mobile and battery-based devices.

\section{REFERENCES}

[1] A. Sendonaris, E. Erkip, and B. Aazhang, "User cooperation diversity. part I and II,” IEEE Trans. Comm., vol. 51, no. 11, pp. 1927-1948, Nov. 2003.

[2] P. A. Anghel, G. Leu, and M. Kaveh, "Multi-user space-time coding in cooperative networks," in Proc. International Conference on Acoustics, Speech and Signal Processing, 2003.

[3] S. S. Ikki, and M. H. Ahmed, "Performance analysis of adaptive decodeand-forward cooperative diversity networks with best-relay selection," IEEE Trans. Comm., vol. 58, no. 1, pp. 68-72, Jan. 2010.

[4] A. Nosratinia, and A. Hedayat, "Cooperative Communication in wireless networks," IEEE Comm. Mag., Oct. 2004.

[5] A. Bansal, M. Bhatnagar and A. Hjørungnes, "Decoding and performance bound of demodulate-and-forward based distributed Alamouti STBC," IEEE Trans. Comm., vol. 12, no. 2, pp. 702-713, Feb. 2013.

[6] C. Hucher, G. R-B Othman, and J-C. Belfiore, "AF and DF protocols based on Alamouti ST code," in Proc. IEEE International Symposium on Information Theory, Jun. 2007.

[7] S. Han, C. Tellambura, and T. Cui, "SNR-dependent radius control sphere detection for MIMO systems and relay networks," Trans. Emerging Tel. Tech. Wiley Online Library, 2013.

[8] K. K. Amiri, M. Wu, J. R. Cavallaro, and J. Lilleberg, "Cooperative partial detection using MIMO relays," IEEE Trans. Comm., vol. 12, no. 2, pp. 5039-5049, Oct. 2011.

[9] A.A.A. El-Banna, M. Elsabrouty, and A. Abdelrhman, "Low complexity adaptive k-best sphere decoder for2x1 MISO DVB-T2," in Proc. Int. Symp. Wirl. Comm. Sys., Aug. 2013.

[10] Y. Cho et al., MIMO-OFDM wireless communications with MATLAB, 1st ed. , chapter 10, John Wiley \& Sons (Asia) Pte Ltd, 2010. 